Gastrointestinale somatoforme Störungen

Larissa Hetterich, Stephan Zipfel, Andreas Stengel

\begin{abstract}
Somatoforme Störungen im Magen-Darm-Trakt gehen mit einem hohen Leidensdruck für die Patienten einher. Oftmals fällt die Diagnose schwer bzw. wird sie erst spät gestellt. Der Artikel möchte ein klareres Bild dieser Krankheitsbilder zeichnen und die Diagnostik und Therapiemöglichkeiten genauer beleuchten. Die neu überarbeitete AWMF-Leitlinie von 2018 berücksichtigt die somatoformen gastrointestinalen Störungen [1].
\end{abstract}

\section{FALLBEISPIEL TEIL 1}

Die 33-jährige Patientin lebt mit ihrem Mann (seit 12 Jahren verheiratet) und den beiden Kindern (Sohn 10 J., Tochter 4J.) zusammen. Sie kümmert sich um den Haushalt, ihr Mann arbeitet im Außendienst bei einer Technikfirma. Sie hat früher bei einer Behörde im Büro gearbeitet; seit der Geburt der Kinder arbeitet sie nicht mehr. Sie würde gern wieder arbeiten, traut sich dies aufgrund der Beschwerden jedoch aktuell nicht zu und beschreibt eine deutliche Einschränkung ihres Alltags durch die Beschwerden.

Ihr Vater hat vor 3 Jahren die Diagnose Magenkarzinom bekommen, vor wenigen Wochen ist er verstorben. Das war für alle eine schwierige Zeit.

Die Patientin hat seit etwa 10 Jahren Beschwerden im Magen-Darm-Bereich. Begonnen hat alles mit einem Darminfekt und der folgenden Antibiotikatherapie. Die Beschwerden sind in den letzten 2 Jahren deutlich progredient, besonders im Sommer dieses Jahres ist es sehr schlimm gewesen. Sie bemerkt störende Darmgeräusche, Meteorismus, rezidivierende Übelkeit ohne Erbrechen, breiige Stühle (2-8/d) und abdominelle Schmerzen; die Beschwerden bestehen nur tagsüber.

\section{Einleitung}

Somatoforme Störungen sind Krankheitsbilder, welche mit einer hohen Prävalenz von bis zu 15\% einhergehen [2] und somit eine sozioökonomische Relevanz haben. Patienten mit einer somatoformen Störung haben bis zu 9-fach höhere Behandlungskosten als die durchschnittliche Bevölkerung [3]. Ungefähr $62 \%$ der Betroffenen suchen einen Arzt auf und beinahe $41 \%$ aller Patienten einer gastroenterologischen Praxis haben eine funktionelle gastrointestinale Störung [4]. Für den Einzelnen bedeutet eine solche Störung oftmals einen hohen
Leidensdruck, verbunden mit einer deutlichen Einschränkung der Lebensqualität [5]. Die International Classification of Diseases (ICD-10) definiert somatoforme Störungen als Krankheitsbilder mit rezidivierend dargebotenen körperlichen Symptomen. Die betroffenen Patienten suchen wiederholt meist unterschiedliche Ärzte auf und fordern hartnäckig medizinische Untersuchungen und Diagnostik ein. Dieses Verhalten bleibt meist auch nach negativen Vorbefunden und der wiederholten ärztlichen Versicherung, dass diese Beschwerden nicht durch die körperlichen Befunde erklärbar seien, bestehen. Eventuell vorhandene körperliche Befunde können meist das Ausmaß der Beschwerden nicht erklären.

\section{Merke}

Patienten mit einer somatoformen Störung weisen einen enormen Leidensdruck durch ihre Erkrankung und Symptome auf und sind meist stark im Alltag eingeschränkt.

Oftmals gibt es eine zeitliche Korrelation zu Schweregrad und Auftreten der Beschwerden und belastenden Lebensereignissen.

\section{PRAXISTIPP}

Patienten mit somatoformen autonomen Störungen fordern oftmals hartnäckig diagnostische Maßnahmen ein und äußern einen deutlichen Leidensdruck. Eventuell vorhandene körperliche Befunde erklären das Ausmaß der Beschwerden nicht.

Somatoforme Störungen können eingeteilt werden in:

- Somatisierungsstörung (F45.0)

- Undifferenzierte Somatisierungsstörung (F45.1)

- Hypochondrische Störung (F45.2)

- Somatoforme autonome Funktionsstörung (F45.3)

- Anhaltende Schmerzstörung (F45.4) 
- Sonstige somatoforme Störungen (F45.8)

- Somatoforme Störung, nicht näher bezeichnet (F45.9)

Bezogen auf den Gastrointestinaltrakt spielt vor allem die somatoforme autonome Funktionsstörung eine wichtige Rolle. Die somatoformen autonomen Funktionsstörungen manifestieren sich symptomatisch v.a. in einem vegetativ innervierten Organ. Typische Symptome im Gastrointestinaltrakt sind:

- Stuhldrang

- Diarrhö

- Verstopfung

- Blähungen

- Flatulenzen

- Schmerzen

Im ICD-10 sind das Reizdarmsyndrom und die funktionelle Dyspepsie über die Funktionsstörung definiert und organbezogen zu finden („Sonstige Erkrankungen des Darms“ und „Krankheiten des Ösophagus, des Magens und des Duodenums“). Sollten bei diesen funktionellen Erkrankungen die o.g. Merkmale hinzukommen, kann von einer somatoformen autonomen Funktionsstörung (in diesem Falle des oberen oder unteren Verdauungssystems) gesprochen werden.

\section{BEGRIFFSERKLÄRUNG}

Generell wird in der Gastroenterologie häufiger von der funktionellen Dyspepsie und dem Reizdarmsyndrom gesprochen, in der Psychosomatik häufigerwenn die Diagnosekriterien erfüllt sind-auf die somatoforme autonome Funktionsstörung Bezug genommen. Auch wenn beide Bezeichnungen nicht synonym verwendet werden sollten, so ist doch eine große Überlappung gegeben.

\section{Definitionen und Symptome}

\section{Funktionelle Dyspepsie (FD)}

Bei der funktionellen Dyspepsie handelt es sich um eine funktionelle Störung des oberen Verdauungstraktes. Anhand der Rom-IV-Kriterien von 2016 (und in Ermangelung einer deutschen Leitlinie) lassen sich 3 Subtypen unterscheiden:

- das epigastrische Schmerz-Syndrom (epigastric pain syndrome, EPS),

- das postprandiale Distress-Syndrom (postprandial distress syndrome, PDS) und ein

- Mischtyp von EPS und PDS.

Merke

Die funktionelle Dyspepsie lässt sich aufgrund der unterschiedlichen Hauptbeschwerden in die 3
Subtypen unterscheiden: epigastrisches Schmerz-Syndrom, postprandiales Distress-Syndrom und Mischtyp.

Es gibt verschiedene Symptome, die für die Diagnosestellung der funktionellen Dyspepsie entscheidend sind: postprandiales Völlegefühl, frühe Sättigung, epigastrische Schmerzen und epigastrisches Brennen.

Die Diagnose funktionelle Dyspepsie erfolgt, wenn mindestens eines der oben genannten Symptome für 3 Monate anhält und der Beginn der Symptome mindestens 6 Monate vor Diagnosestellung liegt. Beim PDS dominieren Beschwerden wie postprandiale Sättigung und/oder frühe Sättigung, welche die Alltagsaktivitäten oder auch das vollständige Beenden einer Mahlzeit mehr als 3-mal pro Woche einschränken. Im Gegensatz dazu sind die Hauptbeschwerden beim EPS epigastrischer Schmerz und / oder epigastrisches Brennen. Diese Symptome müssen zur Diagnosestellung die Alltagsaktivitäten einschränken und mindestens einmal pro Woche auftreten [6].

\section{DIAGNOSEKRITERIEN FD}

Zur Diagnosestellung der funktionellen Dyspepsie gehört:

- Eines der 3 Kardinalsymptome hält für 3 Monate an.

- Beginn der Symptome mindestens 6 Monate vor Diagnosestellung.

- Einschränkung der Alltagsaktivitäten.

\section{Reizdarmsyndrom (RDS)}

Das Reizdarmsyndrom als funktionelle Störung des unteren Verdauungssystems kann mit unterschiedlichen Symptomen einhergehen. Charakteristisch sind vor allem abdominelle Schmerzen, die häufig rezidivierend und chronisch sind, sowie veränderte Stuhlganggewohnheiten, wobei sowohl Obstipation als auch Diarrhö vorkommen. Ebenfalls klagen Patienten über Blähungen und abdominelle Distension. Die Symptome sind oftmals verbunden mit einer enormen Einschränkung der Lebensqualität und einem hohen Leidensdruck, ähnlich wie bei chronischen somatischen Erkrankungen wie dem Diabetes mellitus, der koronaren Herzkrankheit oder einer chronischen Niereninsuffizienz. In Deutschland gilt noch die S3-Leitlinie von 2011 [7]; sie ist derzeit in Überarbeitung. Ein Reizdarmsyndrom liegt demnach dann vor, wenn folgende 3 Kriterien erfüllt sind:

- Es bestehen chronische, länger als 3 Monate anhaltende, auf den Darm bezogene Beschwerden (z. B. Bauchschmerzen, Blähungen), die für gewöhnlich mit Stuhlgangveränderungen einhergehen (aber nicht müssen).

- Es liegt eine durch die Beschwerden begründete relevante Beeinträchtigung der Lebensqualität vor. 
- Es liegen keine für andere Krankheitsbilder charakteristische Veränderungen vor, die wahrscheinlich für die Symptome verantwortlich sind.

In den USA gelten seit 2016 für die Diagnosestellung eines Reizdarmsyndroms die Rom-IV-Kriterien. Diese setzen eine Symptomdauer von mindestens 6 Monaten vor der Diagnosestellung voraus. Leitsymptom sind hierbei die rezidivierenden abdominellen Schmerzen. Diese müssen in den letzten 3 Monaten mindestens einmal pro Woche aufgetreten sein. Zusätzlich müssen diese Schmerzen mit mindestens 2 der 3 folgenden Kriterien zusammen aufgetreten sein:

- assoziiert mit der Defäkation,

- assoziiert mit der Veränderung der Stuhlfrequenz und / oder

- assoziiert mit einer Veränderung der Stuhlkonsistenz.

Wie bei den vorherigen ROM-III-Kriterien gibt es auch bei den Rom IV-Kriterien die Unterteilung in verschiedene Untertypen des Reizdarmsyndroms. Diese werden anhand der Stuhlgewohnheiten definiert:

- RDS-D (diarrhea): > $25 \%$ der Stühle sind flüssig, ohne feste Bestandteile, $<25 \%$ sind einzelne harte Klumpen.

- RDS-C (constipation): > $25 \%$ der Stühle sind einzelne harte Klumpen, $<25 \%$ flüssig, ohne feste Bestandteile.

- RDS-M (mixed): >25\% der Stühle sind flüssig, ohne feste Bestandteile und > $25 \%$ sind einzelne harte Klumpen.

- RDS-U (unclassified): nicht eindeutig zuzuordnen.

\section{Merke \\ Die Unterteilung des Reizdarmsyndroms mittels der Rom-IV-Kriterien in 4 verschiedene Subtypen erfolgt anhand der Stuhlgewohnheiten.}

Insgesamt beziehen sich die Rom-IV-Kriterien vorrangig auf ein abnormes Stuhlverhalten. Für die Klassifikation dieses Stuhlverhaltens wird die Bristol-Stuhlformen-Skala verwendet. Die Patienten sollten dies über mindestens 2 Wochen in einem Tagebuch dokumentieren. Weitere Begleitsymptome sind häufig, z.B. Stuhldrang, Gefühl der unvollständigen Darmentleerung, Stuhlgang mit Schleimbeimischung oder Druck- und Völlegefühl nach den Mahlzeiten. Da diese Symptome jedoch unspezifisch sind und auch bei anderen Krankheiten häufiger vorkommen, werden sie nicht alleinig zur Diagnosestellung herangezogen.

Dennoch sind dies die Symptome, mit denen sich die Patienten beim Arzt vorstellen und die letztlich-vor allem in Abwesenheit von bisher verfügbaren Bio-/ Psycho-Markern - zur Diagnosestellung führen. Für die Diagnose des Reizdarmsyndroms spricht auch das Fehlen von Warnhinweisen oder Risikofaktoren, wie z. B. eine positive Familienanamnese für ein Kolonkarzinom, chronisch-entzündliche Darmerkrankungen, Zöliakie, Alter > 50, Fieber, nächtliche Diarrhö, Anämie, Hämatochezie und ein ungewollter Gewichtsverlust von $>10 \%$ in den letzten 3 Monaten. Sind diese Warnhinweise nicht vorhanden, wird die Diagnose eines Reizdarmsyndroms wahrscheinlicher [7].

\section{Prävalenz}

Die Prävalenz der funktionellen Dyspepsie beträgt in westlichen Ländern 10-40\%, während in asiatischen Ländern eine Prävalenz von 5-30\% beschrieben wurde [6]. Die Prävalenz des Reizdarmsyndroms liegt weltweit bei ca. 1-45\% (gepoolt bei ca. 11,2\%) [8], in Deutschland bei etwa $15-22 \%$ [9].

Merke

In Deutschland leiden etwa 10-40\% der Bevölkerung an einer funktionellen Dyspepsie und 15-22\% an einem Reizdarmsyndrom.

Es ist weiterhin bekannt, dass es eine Assoziation zwischen der funktionellen Dyspepsie und dem Reizdarmsyndrom gibt. So bekommen Patienten mit einer funktionellen Dyspepsie im Verlauf von Jahren häufig Reizdarmsymptome, auch umgekehrt ist ein solcher Symptomshift / eine Symptomerweiterung beschrieben.

\section{Komorbiditäten}

\section{FALLBEISPIEL TEIL 2}

Frau $\mathrm{T}$. hat zudem eine rezidivierende Stimmungsveränderung bemerkt (erstmals vor ca. 9 Jahren), dies ist von Phasen mit normaler Stimmung unterbrochen. Aktuell ist die Stimmung deutlich gedrückt, sie fühlt sich freudlos und antriebsarm. Sie muss rasch weinen.

Psychopathologischer Befund

Psychischer Befund: Bewusstsein/Vigilanz: keine quantitativen oder qualitativen Bewusstseinsänderungen; Aufmerksamkeit/Gedächtnis: vermindert, keine amnestischen Defizite; Orientierung: zeitlich, örtlich, situativ und personell orientiert; Wahrnehmung: keine qualitativen und quantitativen Wahrnehmungsänderungen; Denken: Grübeln, Gedankenkreisen, negative Zukunftsgedanken, gesundheitsbezogene Sorgen; Affektivität: vermindert schwingungsfähig, Stimmung deutlich gedrückt, freudlos; Antrieb / Psychomotorik: Antrieb und Leistungsfähigkeit vermindert, innere Unruhe, Durchschlafstörungen, Appetit vermindert; Ich-Erleben: keine Störungen des Einheitserlebens des Ichs, keine Veränderungen der IchUmwelt-Grenze; Intelligenz: kein Hinweis auf angeborene oder erworbene Intelligenzminderung; akute 
Suizidalität: rezidivierende Todesphantasien, aktuell distanziert.

In der Zusammenschau liegt somit eine komorbide rezidivierende depressive Störung mit aktuell schwergradiger Symptomatik vor.

Patienten mit somatoformen autonomen Funktionsstörungen haben in 50-70\% der Fälle zusätzliche Beschwerden, wobei Komorbiditäten sowohl somatoform, somatisch oder psychisch sein können. Die Überlappung von unterschiedlichen somatoformen Störungen ist relativ häufig und macht die Behandlung anspruchsvoller [10]. Die aktuell in Vorbereitung begriffene ICD-11 greift die Häufigkeit der (v. a. somatoformen) Komorbiditäten auf und fasst diese Erkrankungen als „bodily distress disorders“ zusammen. Psychische Komorbiditäten sind bei Patienten mit Reizdarmsyndrom in 50-94\% der Fälle berichtet worden, wobei sich die Spannbreite am ehesten mit den unterschiedlichen Erfassungszentren erklären lässt (primär vs. tertiär, wobei die letztgenannten Patienten oftmals durch einen schwereren und prolongierten Krankheitsverlauf gekennzeichnet sind). Hierbei sind vor allem Angststörungen (30-50\%), Depressionen (70\%) aber auch Essstörungen zu nennen. Es konnte gezeigt werden, dass diese Komorbiditäten einen maßgeblichen Einfluss auf den Therapieverlauf haben [11]. Auch weitere Krankheitsbilder mit somatoformer Komponente sind komorbid bei ca. 50 \% der Patienten anzutreffen, wie das Fibromyalgiesyndrom, die chronische Fatigue, die überaktive Blase, das chronische Beckenschmerzsyndrom und Spannungskopfschmerzen. An somatischen Erkrankungen, welche mit dem Reizdarmsyndrom assoziiert sind, ist z. B. die gastroösophageale Refluxkrankheit nennen.

\section{Merke}

Das Vorhandensein einer oder mehrerer Komorbiditäten schränkt die Lebensqualität der Betroffenen zusätzlich deutlich ein.

Bereits frühzeitig konnte gezeigt werden, dass Distress zu einer Verschlimmerung der gastrointestinalen Symptome führt. Dabei handelt es sich oftmals um einen Teufelskreis, da durch die gastrointestinalen Beschwerden noch mehr Disstress entsteht und die Symptome daraufhin oftmals perpetuieren und persistieren können.

Auch bei Patienten mit funktioneller Dyspepsie finden sich häufig Komorbiditäten. Angststörungen und Depressionen kommen in ähnlicher Häufigkeit vor, komorbide Essstörungen sind jedoch deutlich häufiger anzutreffen als beim Reizdarmsyndrom. Inwiefern die psychische Komorbidität an der Entstehung der funktionellen Dyspepsie (mit)beteiligt ist, ist noch nicht abschließend geklärt, da sowohl die psychische Erkrankung die Entstehung einer funktionellen Dyspepsie begünstigen kann, jedoch auch die funktionelle Dyspepsie ein prädisponierender Faktor für die Entstehung einer weiteren Erkrankung sein kann [6].

\section{Merke}

Patienten mit somatoformen autonomen Funktionsstörungen des Gastrointestinaltraktes haben häufig Komorbiditäten. Diese können psychischer, somatischer oder somatoformer Natur sein.

\section{Pathophysiologie}

Die Pathogenese der somatoformen autonomen Funktionsstörungen des Verdauungssystems ist am besten über das biopsychosoziale Modell zu erklären (Abb. 1). Dabei spielen biologische, psychische und soziale Faktoren in unterschiedlicher Konstellation eine Rolle. Hierbei können sowohl genetische Faktoren wie auch die Umwelt spätere Persönlichkeitsmerkmale beeinflussen. Zu den Persönlichkeitsmerkmalen gehören auch Bewältigungsstrategien, die dann pathogenetisch bedeutsam werden, wenn diese nicht mehr ausreichen. Für das Reizdarmsyndrom v. a. bedeutsam sind folgende Faktoren: frühere Lebenserfahrungen, Verhalten der Eltern, soziales Lernen, Kultur, Traumata, Infektionen, Stress und das Ausmaß der Unterstützung, die ein Individuum in seinem Leben erhalten hat. Diese Faktoren spielen auch bei der Entstehung der funktionellen Dyspepsie eine wichtige Rolle. Zusätzlich gibt es weitere Risikofaktoren, die eine Krankheitsentstehung erleichtern, wie z. B. eine Reisediarrhö, die Einnahme von Antibiotika oder nichtsteroidalen Antirheumatika, Rauchen und Übergewicht.

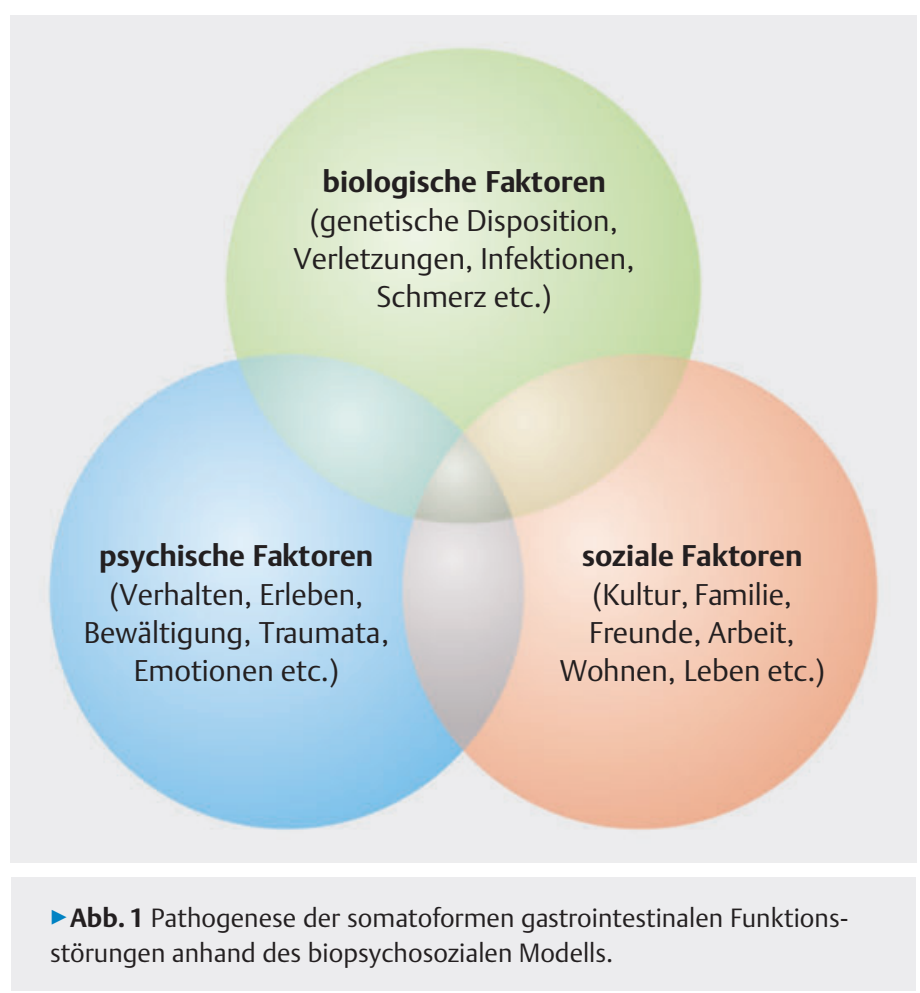




\section{BIOPSYCHOSOZIALES MODELL}

Das biopsychosoziale Modell enthält Faktoren der Psyche, der Biologie und des sozialen Umfeldes und spielt eine wesentliche Rolle in der Pathophysiologie von somatoformen autonomen Funktionsstörungen des Gastrointestinaltraktes.

Als besonderer Risikofaktor für die Entwicklung von somatoformen autonomen Funktionsstörungen des Verdauungssystems konnten negative Lebensereignisse (sogenannte „Life Events“) beschrieben werden; hier ist das Erleben z. B. von Missbrauch oder Traumatisierungen als Risikofaktor zu nennen [12].

Ein weiterer wichtiger Baustein in der Pathophysiologie somatoformer autonomer Funktionsstörungen des Verdauungssystems ist die Darm-Gehirn-Achse, in letzter Zeit noch erweitert um das Darmmikrobiom. Allerdings besteht hier noch erheblicher Forschungsbedarf, um die Zusammensetzung eines „gesunden“ Mikrobioms besser zu verstehen.

Merke

Die Darm-Gehirn-Achse ist ein bidirektionales Kommunikationssystem zwischen dem zentralen Nervensystem (ZNS) und dem Darm.

Über afferente Nervenfasern gelangen Informationen aus dem Gastrointestinaltrakt zum ZNS, werden dort verarbeitet und weiter verknüpft-beispielsweise mit emotionsverarbeitenden Gehirnarealen - und über efferente Leitungsbahnen zurück an den Darm geschickt, wodurch die Darmfunktion moduliert werden kann. Um eine Veränderung dieses Kommunikationssystems bei somatoformen autonomen Funktionsstörungen des Verdauungssystems hervorzuheben, wird von diesen in den Rom-IV-Kriterien auch als Störung der „Gut-Brain Interaction“ gesprochen. Diese Störung kann Auswirkungen auf Darmmotilität, intestinale Immunantwort, oder intestinale Permeabilität haben. Durch die erleichterte Durchgängigkeit können Entzündungszellen den Darm durchdringen und zu einer lokalen Entzündungsreaktion führen, welche wiederum eine viszerale Hypersensitivität begünstigt. So kommt es bei der funktionellen Dyspepsie sowohl durch die Entzündungsreaktion wie auch durch die pathologischen Antworten auf chemische und mechanische Reize zu einer viszeralen Hypersensitivität, die auch beim Reizdarmsyndrom beobachtet werden kann [13].

\section{FAZIT FÜR DIE PRAXIS}

Eine Störung in der (Mikrobiom)-Darm-Gehirn-Achse wirkt sich auf Darmmotilität, Immunantwort und intestinale Permeabilität aus und ist somit ebenfalls ein wichtiger Baustein in der Entstehung des Reizdarmsyndroms und der funktionellen Dyspepsie.

\section{Diagnostik}

\section{FALLBEISPIEL TEIL 3}

Die Patientin hat im Zuge der Beschwerden eine Vielzahl von verschiedenen Ärzten aufgesucht. Die durchgeführte organopathologische Diagnostik (körperliche Untersuchung, Labor, mehrfach Ösophagogastroduodenoskopie, einmalig mit tiefen Duodenalbiopsien und mehrfach Koloskopie, einmalig inklusive Stufenbiopsien, Sonografie des Abdomens, gynäkologischer Ultraschall) hat keinen wegweisenden Befund erbracht. In den Atemtests sind eine Laktoseintoleranz und eine Fruktosemalabsorption festgestellt worden, die entsprechende Diät hat jedoch keine deutliche und anhaltende Beschwerdebesserung erbracht. Eine Rückversicherung an die Patientin, dass es keine (rein) körperliche Ursache der Beschwerden gibt, erbrachte nur eine sehr kurzfristige Beruhigung.

Zusammenfassend kann somit die Diagnose einer somatoformen autonomen Funktionsstörung des oberen und unteren Verdauungssystems vergeben werden. Wie oben beschrieben, liegt eine komorbide rezidivierende depressive Störung mit aktuell schwergradiger Symptomatik vor.

Da es noch keine deutsche Leitlinie zur funktionellen Dyspepsie gibt, hat die Deutsche Gesellschaft für Neurogastroenterologie und Motilität lediglich Empfehlungen zur Diagnostik bereitgestellt. Entsprechende Warnsymptome, die sich aus der Anamnese ergeben können, sind Gewichtsabnahme, Dysphagie, Odynophagie, rezidivierendes Erbrechen, Appetitlosigkeit, Anämie / Zeichen der gastrointestinalen Blutung, Fieber, höheres Lebensalter (50 + / - 5 Jahre), familiäre Belastung mit gastrointestinalen Karzinomen und eine kurze Anamnese. Diese Warnhinweise sollten nicht übersehen werden und lassen primär an andere Krankheitsbilder denken, welche dann bestätigt oder ausgeschlossen werden sollten.

Aus der Anamnese sollte auch der entsprechende Subtyp erfasst werden. Die Anamnese alleine ist jedoch nicht ausreichend und weitere Diagnostik ist zwingend notwendig. Die Deutsche Gesellschaft für Neurogastroenterologie und Motilität empfiehlt eine körperliche Untersuchung, ein Basislabor (kleines Blutbild, CRP / BSG, Gamma-GT, GOT/GPT, Kreatinin, Lipase), eine Abdomen-Sonografie und eine Ösophago-Gastro-Duodenoskopie (ÖGD). Die Durchführung eines abdominellen Ultraschalls kann hilfreich sein, um eine biliäre Genese 
wie auch eine maligne Erkrankung auszuschließen. Ebenso sollten mindestens zwei unterschiedliche Testverfahren für den Nachweis von Helicobacter pylori durchgeführt werden. Damit kann eine durch Helicobacter pylori ausgelöste Dyspepsie ausgeschlossen werden; diese wäre dann durch eine Eradikation behandelbar [6]. Auch weitere Ausschlussdiagnostik wie eine 24h-pHMetrie oder Dünndarmmanometrie ist möglich, aber nicht zwingend notwendig.

\section{DIAGNOSTIK BEI FUNKTIONELLER DYSPEPSIE}

- Anamnese mit Warnsymptomen (welche primär an andere Erkrankung denken lassen)

- körperliche Untersuchung

- Basislabor

- Abdomen-Sonografie

- Ösophago-Gastro-Duodenoskopie

- Test auf Helicobacter pylori

Zur Diagnosestellung des Reizdarmsyndroms sollten gemäß S3-Leitlinie nach einer ausführlichen Anamnese eine körperliche Untersuchung (inklusive digitalrektaler Untersuchung) sowie eine Laboruntersuchung erfolgen. Auch eine Stuhluntersuchung zum Ausschluss pathogener Keime inklusive Parasiten ist sinnvoll. Obligat sind weiterhin ein abdomineller Ultraschall sowie eine gynäkologische Untersuchung bei Frauen. Die Durchführung einer Endoskopie (obere und untere) sollte bei entsprechender Symptomatik zum Ausschluss einer Zöliakie sowie einer chronisch entzündlichen Darmerkrankung erfolgen, bei Durchfallsymptomatik ist an die Entnahme von Stufenbiopsien zum Ausschluss einer mikroskopischen Kolitis zu denken. Auch die Durchführung von $\mathrm{H}_{2}$ Atemtests sollte zum Ausschluss einer Laktoseintoleranz und Fruktosemalabsorption erfolgen, da diese Unverträglichkeiten leicht behandelbar sind. Die Ausschlussdiagnostik ist gegebenenfalls anhand der Symptomatik der Patienten zu erweitern [7].

\footnotetext{
DIAGNOSTIK BEI REIZDARMSYNDROM

- Anamnese mit Warnsymptomen (welche primär an andere Erkrankung denken lassen)

- körperliche Untersuchung inklusive digital-rektaler Untersuchung

- gynäkologische Untersuchung

- Basislabor

- Stuhluntersuchung auf pathogene Keime

- Sonografie des Abdomens

- Gastro- und Koloskopie

- $\mathrm{H}_{2}$-Atemtests
}

Die Diagnose einer somatoformen autonomen Funktionsstörung kann dann gestellt werden, wenn in den vorliegenden diagnostischen Untersuchungen keine pathologischen Veränderungen sichtbar sind, die die Symptome der Patienten ausreichend erklären und zusätzlich die Positivkriterien einer somatoformen Störung vorliegen (für das Reizdarmsyndrom allein nicht zwingend notwendig). Es sollte darauf geachtet werden, keine unnötige und/oder wiederholende Diagnostik anzuwenden, da dadurch die Patienten in ihrem (oftmals rein somatischen) Krankheitskonzept bestärkt würden und eine weitere Einforderung von-womöglich immer invasiverer-Diagnostik erfolgen würde.

\section{Merke}

Ein wichtiger Schritt ist die Übermittlung der Diagnose, welcher bereits Teil der Therapie im Sinne der Psychoedukation ist.

\section{Therapie}

\section{FALLBEISPIEL TEIL 4}

Bei der Erstvorstellung nimmt die Patientin Heilerde und Simethicon ein. Dies verbessert die Beschwerden nicht. Eine psychotherapeutische Behandlung hat bisher nicht stattgefunden, von einer gastroenterologischen Rehamaßnahme hat sie nur minimal profitiert. Die von uns angebotene bedarfsweise Behandlung mit Loperamid verschafft Linderung in als belastend erlebten Situationen, die symptomatische Therapie mit Mebeverin und Butylscopolamin wird als nicht hilfreich erlebt. Zur Schmerzreduktion besprechen wir mit der Patientin die Off-Llabel-Therapie mit Amitriptylin (Start mit $12,5 \mathrm{mg}$ zur Nacht, alle 2 Wochen Steigerung auf max. $50 \mathrm{mg}$ zur Nacht). Hierunter reduziert sich die Stuhlfrequenz und verbessert sich die Stuhlkonsistenz. Dennoch muss sie weiterhin 3- bis 4-mal auf die Toilette, hat weiterhin Meteorismus und Schmerzen. Die Beschwerden beeinträchtigen sie stark, sie grübelt viel darüber nach und zieht sich zunehmend sozial zurück.

In Anbetracht der Komorbidität und der Schwere der Symptomatik sehen wir zur Verhinderung einer weiteren Progredienz und Chronifizierung die Indikation zur stationären psychosomatischen Behandlung. Dies wird von der Patientin begrüßt. Folgende Therapieziele werden zu Beginn von der Patientin formuliert: Strategien zum Umgang bezüglich der aktuellen Symptomatik, Verbesserung der Lebensqualität, Stimmungsstabilisierung und Bewältigung der angstbesetzten Zustände (Sorge um andere, Sorge um sich selbst).

Die Patientin nimmt am Therapieprogramm für somatoforme Erkrankungen teil, bestehend aus Einzel- und 
Gruppentherapien, Chef- und Oberarztvisiten, einer interpersonellen Gruppe zur Depressionsbehandlung, Musiktherapie, Bewegungstherapie, Physiotherapie und einer speziellen Gruppe zum Erlernen von Entspannungstechniken.

Frau T. kann sich nach einer Eingewöhnungszeit gut in den Stationsalltag und die Patientengemeinschaft integrieren. Sie setzt sich mit ihrer biografischen Entwicklung und bisherigen Beziehungserfahrungen auseinander, die Einfluss auf die Krankheitsentstehung und vor allem -aufrechterhaltung haben. Die geregelte Tagesstruktur und das Gefühl, Hilfe und Unterstützung für ihre Beschwerden zu bekommen, die Wahrnehmung sozialer Kontakte und Durchführung von sozialen Aktivitäten trotz Beschwerden führen zu einer signifikanten Verbesserung der Stimmung wie auch der Magen- und Darmbeschwerden. Insgesamt kann die Patientin ein verbessertes Krankheitsverständnis mit psychosomatischen Aspekten entwickeln und in ersten Schritten einen funktionaleren Umgang mit den Beschwerden einüben. Die Bauchbeschwerden sind weiterhin vorhanden, aber in deutlich geringerem und wechselndem Ausmaß. Ebenso hat sich die Stimmung wieder stabilisiert, vor allem waren hierfür soziale Kontakte und Aktivitäten hilfreich. Die Patientin möchte zur weiteren Stabilisierung eine ambulante Psychotherapie aufnehmen.

Die therapeutischen Ziele in der Behandlung des Reizdarmsyndroms und der funktionellen Dyspepsie sind vorrangig, die Symptome zu lindern und damit die Lebensqualität der Patienten zu verbessern. Für beide funktionelle Störungen gibt es nicht nur eine Therapiemöglichkeit, sondern viele verschiedene Ansätze. Zuallererst sollte den Patienten ihr Krankheitsbild verständlich vermittelt werden, damit sie aktiv in die Behandlung einbezogen werden können. Da die Patienten oftmals viele Arztbesuche und Diagnostik hinter sich haben, ist es wichtig, eine (möglichst) stabile Arzt-Patienten-Beziehung aufzubauen.

\section{PRAXISTIPP}

Um ein besseres Verständnis für die subjektive Auffassung der Patienten über ihre Krankheit zu bekommen, empfiehlt sich, spezielle Fragebögen anzuwenden, wie z. B. den Illness Perception Questionnaire.

Ebenso ist es wichtig, dass die unterschiedlichen krankheitsauslösenden Faktoren eruiert werden. Dazu gehört, dass die Assoziation der Symptome mit belastenden Faktoren wie Stress eine wichtige Rolle spielt. Regelmäßige Termine nehmen den Patienten die Angst, genauso wie die Teilnahme an Selbsthilfegruppen oder Gespräche den Verlauf positiv beeinflussen können. Im Folgenden werden die unterschiedlichen Therapiemöglichkeiten vorgestellt.

\section{Medikamentöse Therapie}

Eine medikamentöse Therapie sollte symptomorientiert sein und vor allem beim Reizdarmsyndrom mit moderater Symptomschwere angewendet werden (s. - Tab. 1). Wenn die Patienten nicht innerhalb von 3 Monaten ansprechen, sollte man eine Umstellung der Medikation erwägen. Eine Möglichkeit ist die Kombination verschiedener Substanzen.

Merke

Generell sollte die medikamentöse Therapie gemeinsam mit diätetischen Maßnahmen oder-bei schwereren Verlaufsformen - psychotherapeutischen Verfahren durchgeführt werden.

Bei der medikamentösen Therapie ist es wichtig, eine Unterscheidung der verschiedenen Subtypen des Reizdarmsyndroms vorzunehmen. Entsprechend werden für das RDS-D Opioid-Antagonisten (z. B. Loperamid) oder $5 \mathrm{HT}_{3}$-Antagonisten (z. B. Ondansetron) eingesetzt ( Tab.1). Dies führt zu einer erhöhten Transitzeit des Kolons. Beim RDS-C dominieren Laxanzien wie Macrogol. Zusätzlich werden oft Spasmolytika wie Butylscopolamin verschrieben, die entsprechend gegen die Bauchkrämpfe wirken. Auch die Gabe von Psychopharmaka kann eine Symptomlinderung herbeiführen. Eine wichtige Rolle spielen hierbei trizyklische Antidepressiva, selektive SerotoninWiederaufnahmehemmer und Serotonin-NoradrenalinWiederaufnahmehemmer. Die trizyklischen Antidepressiva haben den Vorteil, dass sie auch gut auf viszerale Schmerzen wirken, während die selektiven Serotonin-Wiederaufnahmehemmer bei komorbider Angststörung geeignet sind und eine gute Verträglichkeit aufweisen. Da trizyklische Antidepressiva auch die Kolontransitzeit verlängern, werden sie häufig bei Patienten mit RDS-D eingesetzt, während SSRI prokinetisch wirken und somit einen negativen Effekt auf Diarrhö besitzen. Generell werden die trizyklischen Antidepressiva geringer dosiert, wenn sie nur gegen viszerale Schmerzen eingesetzt werden (Off-LabelTherapie). Bei psychischen Komorbiditäten werden die trizyklischen Antidepressiva in höherer (antidepressiver) Dosierung verabreicht.

Bei der funktionellen Dyspepsie werden andere Medikamentengruppen verwendet ( $\triangleright$ Tab. 2). Zum einen kommen Protonenpumpeninhibitoren bei säurebetonter Dyspepsie zum Einsatz. Ebenso können Dopamin-2Rezeptor-Antagonisten wie Domperidon oder Metoclopramid genutzt werden. Auch Simethicon als Entschäumer ist eine oft genutzte Therapie für die funktionelle Dyspepsie. Ebenso sind Serotonin-Agonisten wie auch -Antagonisten eine Therapiemöglichkeit bei der funktionellen Dyspepsie. Auch Phytopharmaka haben ihren Stellenwert [6]. 
\Tab. 1 Zugelassene/verfügbare medikamentöse Therapiemöglichkeiten beim Reizdarmsyndrom.

\begin{tabular}{|c|c|}
\hline Medikament & Gewünschte Wirkung \\
\hline Opioid-Antagonisten (z. B. Loperamid) & Erhöhte Transitzeit des Kolons v. a. beim RDS-D \\
\hline $5 \mathrm{HT}_{3}$-Antagonisten (z.B: Ondasentron) & Erhöhte Transitzeit des Kolons v. a. beim RDS-D, schmerzlindernd \\
\hline Laxanzien (z. B. Macrogol, 5HT 4 -Agonist Prucaloprid) & Gesteigerte Peristaltik und Verflüssigung des Stuhls \\
\hline Spasmolytika (z. B. Butylscopolamin) & $\begin{array}{l}\text { Tonus der glatten Muskulatur wird herabgesetzt, krampflösend, bei } \\
\text { Stuhlunregelmäßigkeiten }\end{array}$ \\
\hline Lösliche Ballaststoffe (z. B. Ispaghula) & Stuhlregulierend, schmerzlindernd, Behandlung beim RDS-D / RDS-O \\
\hline (Probiotika) & Schmerzlindernd, Behandlung v. a. beim RDS-D \\
\hline Phytotherapeutika (z. B. STW 5) & $\begin{array}{l}\text { Spasmolytisch, bei Stuhlunregelmäßigkeiten, Blähungen, Meteorismus, } \\
\text { Flatulenz }\end{array}$ \\
\hline Trizyklische Antidepressiva (z. B. Amitriptylin) & $\begin{array}{l}\text { Verminderung viszeraler Schmerzen, Verlängerung der Kolontransitzeit } \\
\text { (v. a. beim RDS-D), ggf. Verbesserung psychischer Begleitsymptome }\end{array}$ \\
\hline selektive Serotonin-Wiederaufnahmehemmer (z. B. Paroxetin) & $\begin{array}{l}\text { Prokinetisch (v. a. beim RDS-C), Verbesserung psychischer } \\
\text { Begleitsymptome }\end{array}$ \\
\hline Gasbinder (z. B. Simethicon) & (moderate) Verminderung der intestinalen Gasmenge \\
\hline
\end{tabular}

-Tab. 2 Medikamentöse Therapieoptionen bei der funktionellen Dyspepsie.

\begin{tabular}{|c|c|}
\hline Medikament & Gewünschte Wirkung \\
\hline Protonenpumpeninhibitoren (z. B. Pantoprazol) & Hemmung der Magensäuresekretion \\
\hline $\mathrm{D}_{2}$-Rezeptor-Antagonisten (z. B. Domperidon, Metoclopramid) & Antiemetisch, fördern anterograde Peristaltik im Magen \\
\hline Entschäumer (z. B. Simethicon) & Gegen Blähungen, Völlegefühl, Schmerzen im Bauchraum \\
\hline Serotonin-Agonisten (z. B. Sumatriptan, Tandospiron) & $\begin{array}{l}\text { Verändert Magengröße und verringert die durch gastrische Distension } \\
\text { ausgelöste Übelkeit, Anxiolyse, gastrische Akkommodation gesteigert }\end{array}$ \\
\hline $5 \mathrm{HT}_{3}$-Antagonisten (z. B. Ondasentron) & Verbesserung der gastrischen Akkomodation \\
\hline Phytopharmaka (z. B. STW 5) & $\begin{array}{l}\text { Verbesserte gastrische Akkomodation, Verbesserung der Schmerzen, } \\
\text { Symptomlinderung }\end{array}$ \\
\hline
\end{tabular}

\section{Diätetische Maßnahmen}

Eine nicht-medikamentöse Therapieoption für das Reizdarmsyndrom stellt die sogenannte FODMAPDiät dar. Dabei verzichten die Patienten zeitweise auf fermentierbare Oligosaccharide, Disaccharide, Monosaccharide und Polyole (oder reduzieren diese zumindest). Diese Therapie hat in Studien gute Ergebnisse gezeigt und sollte zeitlich begrenzt Anwendung finden. Prinzipiell sollten sich Patienten gesund ernähren und eine fettarme Kost zu sich nehmen. Zusätzlich zu einer gesunden Ernährung ist eine sportliche Betätigung empfohlen. Weiterhin können ausgewählte Probiotika die Symptome des Reizdarmsyndroms verbessern, die Studienlage ist hier jedoch sehr inhomogen, sodass bisher keine klare Empfehlung für den Einsatz von Probiotika gegeben werden kann. Nicht zuletzt kann die Einnahme von Ballaststoffen (v. a. beim RDS-C) symptomlindernd sein.

\section{PRAXISTIPP}

Merke: Eine gesunde Ernährung und sportliche Maßnahmen können wesentlich zur Symptomlinderung führen. Ebenso ist es wichtig, dass der Patient die Nahrungsmittel reduziert und ggf. meidet, die die Symptome verstärkt hervorrufen.

Bei Patienten mit funktioneller Dyspepsie treten die Beschwerden oft nach der Mahlzeiteneinnahme auf, bzw. werden durch diese verstärkt. Auch hier sollten 
die Patienten die Nahrung reduzieren/ggf. meiden, die die entsprechenden Symptome verstärken-eine sehr restriktive Diät sollte jedoch vermieden werden. Ebenso ist der Verzicht auf fettreiche Nahrung und mehr Bewegung eine Möglichkeit, die Symptome zu lindern.

\section{Psychotherapeutische Maßnahmen}

Ein weiterer wichtiger Baustein der Therapie des Reizdarmsyndroms sowie auch der funktionellen Dyspepsie ist die Psychotherapie. Es ist jedoch zu bemerken, dass es mehr Daten zur Psychotherapie beim Reizdarmsyndrom gibt. Anhand des biopsychosozialen Modells zeigt sich der Einfluss von psychischen und sozialen Faktoren auf das Krankheitsausmaß deutlich. Somit nimmt es nicht wunder, dass es mithilfe psychologischer Interventionen möglich ist, Beschwerden zu lindern. Zu den psychotherapeutischen Maßnahmen gehören vor allem

- die Psychoedukation,

- die kognitive Verhaltenstherapie,

- die psychodynamische Psychotherapie und

- die Hypnotherapie.

\section{Psychoedukation}

Wie bei vielen Erkrankungen ist es auch bei funktionellen und somatoformen Störungen des Verdauungssystems essenziell, dass der Patient ausreichend über seine Erkrankung aufgeklärt ist.

\section{Merke}

Für das Aufklärungs- und Beratungsgespräch sollte sich der behandelnde Arzt Zeit lassen.

Viele Patienten haben eine lange Leidensgeschichte und zahlreiche Arztkontakte hinter sich und haben dennoch kein klares Verständnis von Diagnose und Pathogenese. Die entsprechende Vermittlung erfordert Empathie und Verständnis vom behandelnden Arzt, fördert die ArztPatienten-Beziehung und kann bereits eine ggf. geplante Psychotherapie bahnen.

Zur Psychoedukation gehört oftmals auch, die Angehörigen der Patienten miteinzubeziehen, da sich ein verständnisvolles Umfeld förderlich auf den Therapieverlauf auswirkt. Die Psychoedukation kann entweder im Einzelgespräch oder auch in Gruppengesprächen mit anderen Patienten erfolgen. Als Ziel steht vor allem der verbesserte Umgang mit der Krankheit im Vordergrund. Dadurch kann auch die Prognose deutlich verbessert werden.

Trotzdem ist es auch wichtig, die somatischen und körperlichen Beschwerden weiterhin zu beachten und den Patienten nicht auf die psychischen Beschwerden zu reduzieren.

\section{Cave}

Es sollte vermieden werden, vorschnell von Psychogenese zu reden.

\section{Kognitive Verhaltenstherapie}

Die breiteste Studienlage zu psychotherapeutischen Interventionen bei somatoformen autonomen Funktionsstörungen des Verdauungssystems gibt es zur kognitiven Verhaltenstherapie, welche einerseits die Faktoren beleuchtet, die zur Entstehung der Erkrankung geführt haben, und andererseits auch die Faktoren, die zur Aufrechterhaltung beitragen. Bei Patienten mit somatoformen Störungen spielt die Angst vor einer organischen Krankheit eine wichtige Rolle. Die Patienten sind ständig bestrebt, eine organische Ursache zu finden, jeder weitere unauffällige Befund schürt diese oftmals weiter.

Gerade gastrointestinale Symptome können ein großes Unwohlsein verursachen und die Patienten sorgen sich oft vor dem Auftreten der Symptome, wie Diarrhö in bestimmten Situationen. Diese Angst kann zu einem Vermeidungsverhalten führen. Ebenso richten Patienten oftmals ihre komplette Aufmerksamkeit auf die Symptome. Durch die Wahrnehmungsverzerrung reagieren die Patienten häufig übersensibel auf Reize und nehmen ihre Symptome noch stärker war. Es kann hier zum Katastrophisieren kommen: Die Patienten fühlen sich hilflos und unverstanden und nehmen die Symptome als immer gravierender wahr. Dies zieht einen enormen Leidensdruck und eine deutliche Einschränkung der Lebensqualität nach sich. Zusätzlich kann es im sozialen Umfeld zu Problemen kommen - Patienten, die gedanklich zunehmend um ihre subjektiven Beschwerden kreisen, erleben im Verlauf einen sozialen Rückzug bis zur sozialen Isolation. Fehlender sozialer Rückhalt von Familie und Freunden führt wiederum dazu, dass sich Patienten auf ihre Krankheit konzentrieren.

\section{Merke}

Die kognitive Verhaltenstherapie kann den Teufelskreis zwischen Fokussierung auf die Krankheit, fehlendem Rückhalt in der Familie, sozialer Isolation und dadurch weiterer Konzentration auf die Erkrankung durchbrechen.

Dabei sollten Patienten ihre Handlungsweisen verändern, lernen ihre Symptome aktiv zu kontrollieren und damit versuchen ihre Ängste zu reduzieren. Die direkte Konfrontation mit unangenehmen Situationen und das genaue Analysieren dieser Situationen kann den Patienten verschiedene Faktoren aufzeigen, die diese Situationen verschlimmern. Auch die Gedanken, die die Patienten in diesem Moment berichten, werden in die Therapie einbezogen. Im Wesentlichen spielen folgende Punkte eine wichtige Rolle:

- Selbstbeobachtung: Die Patienten sollten sich kritische Fragen stellen und ihr Verhalten beobachten 
und hinterfragen. Auch die genaue Auseinandersetzung mit den Gefühlen spielt eine Rolle.

- Kognitive Umstrukturierung: Die Patienten sollten ihre üblichen Verhaltensweisen vermeiden und somit die kognitiven Verzerrungen verändern.

- Problemlösung: Gemeinsam werden die Probleme beleuchtet und es wird nach Lösungen und Verbesserungen geschaut.

- Konfrontation / Exposition: Die Patienten werden stressauslösenden Situationen ausgesetzt. Dabei sollten die üblichen Muster der Vermeidung und Hypersensibilität vermieden werden und auf die davor ausgearbeiteten Verhaltensweisen zurückgegriffen werden. Diese Konfrontation sollte wiederholt stattfinden, damit die Konditionierung der Angst und Vermeidung reduziert wird und die Patienten sukzessive weniger Angst empfinden.

- Entspannungstechniken: Mit einer geübten Technik sollten die Patienten sich in / vor / nach Stresssituation entspannen können.

\section{Merke}

Eine breite Studienlage zeigt die Wirksamkeit der kognitiven Verhaltenstherapie in Bezug auf somatoforme autonome Funktionsstörungen des

Verdauungssystems.

Die kognitive Verhaltenstherapie verbessert nicht nur die subjektive Symptomwahrnehmung und führt dadurch zu einer gesteigerten Lebensqualität, sondern reduziert auch die Angst und verbessert die Komorbiditäten, wie z. B. die Depression [14].

\section{Psychodynamische Therapie}

Bei der psychodynamischen Therapie besteht ein wesentliches Ziel in der Aufarbeitung von intra- / interpersonellen Konflikten, welche oft maßgeblich an der Symptomausprägung und -schwere beteiligt sind. Die Patienten erlangen im Rahmen der Therapie einen Zugang zu diesen Konflikten und können diese im Rahmen der interpersonellen (meist Einzel-) Therapie bearbeiten. Dabei spielen auch das Erleben zwischenmenschlicher Beziehungen sowie eine bessere Selbstwahrnehmung eine Rolle [15].

Eine Studie zeigte, dass psychodynamische Interventionen einen positiven Einfluss auf die Beschwerden beim Reizdarmsyndrom haben. Im Vergleich zur Kontrollgruppe mit einer rein medikamentösen Therapie erhielt eine Gruppe zusätzlich eine dynamische Psychotherapie. Diese Gruppe zeigte eine signifikante Verbesserung der körperlichen Beschwerden, welche auch ein Jahr später noch nachweisbar war [16].

Eine Metaanalyse untersuchte den Effekt psychodynamischer Interventionen bei Patienten mit Reizdarmsyndrom. Dabei wurden zwei Studien eingeschlossen (insgesamt $n=273$ ) [17]. Hierbei zeigten $45,8 \%$ der Patienten eine
Verbesserung der Symptome im Vergleich zu 29,6\% in der Vergleichsgruppe. Die NNT (Number needed to treat) für psychodynamische Verfahren liegt bei 4 [17].

\section{Hypnotherapie}

Auch die Hypnose stellt eine ernstzunehmende Therapiemöglichkeit bei somatoformen autonomen Funktionsstörungen dar. Bei der Hypnotherapie induziert der Therapeut einen veränderten Bewusstseinszustand bei den Patienten im Sinne einer Heterosuggestion. Die Hypnotherapie beim Reizdarmsyndrom beinhaltet 7 - 12 Sitzungen à 30-60 Minuten über 3 Monate. Ebenso sollten Patienten im Verlauf täglich mithilfe einer CD weiter üben - auch eine kostensparende Version mit einer rein CD-gestützten Intervention wurde bereits bei Kindern untersucht. Während der Therapie ist ein Symptomtagebuch sinnvoll, in welchem die Patienten die Dauer und Intensität der Bauchschmerzen sowie die Stuhlhäufigkeiten, das Befinden, die Dauer der Selbstübungen und weitere Informationen festhalten.

Mehrere Studien haben gezeigt, dass eine Hypnose beim Reizdarmsyndrom, v.a. bei medikamentenrefraktären Verläufen, günstige Effekte haben kann. So haben 24$73 \%$ der Patienten positiv auf eine Hypnose angesprochen. Dies beinhaltet eine Reduktion der gastrointestinalen Symptome sowie eine Verbesserung von Stimmung und Wohlbefinden. Die Hypnotherapie kann bei allen Reizdarm-Subtypen eingesetzt werden (v. a. RDS-C, RDS$M$ und RDS-U) [18]. Für die funktionelle Dyspepsie gibt es bislang nur wenige Daten zur Hypnotherapie, diese sind jedoch vielversprechend.

Eine neue Metaanalyse schloss insgesamt 5 verschiedene Studien über Hypnotherapie mit insgesamt 278 Patienten ein. Dabei zeigten 55,4\% der Patienten eine Verbesserung der Symptome im Vergleich zu 22,6\% in der Kontrollgruppe. Die NNT lag bei 5 [17].

\section{KERNAUSSAGEN}

- Gastrointestinale somatoforme Störungen sind mit einer Prävalenz von 15 \% in Deutschland eine häufige Diagnose.

- Die beiden wichtigsten funktionellen gastrointestinalen Störungen sind die funktionelle Dyspepsie und das Reizdarmsyndrom.

- Das Reizdarmsyndrom wird anhand der deutschen Leitlinie diagnostiziert als chronische, auf den Darm bezogene Beschwerden, welche die Lebensqualität maßgeblich beeinträchtigen. Gemäß den Rom-IV-Kriterien ist eine Subgruppierung anhand der Stuhlgangveränderungen möglich.

- Die funktionelle Dyspepsie beinhaltet gemäß Rom-IV 2 Untertypen: das epigastrische SchmerzSyndrom mit epigastrischem Schmerz / Brennen und das postprandiale Distress-Syndrom mit 
früher postprandialer Sättigung. Weiterhin gibt es noch einen Mischtyp.

- Die Beschwerden werden nicht oder nicht vollständig von einem organopathologischen Befund erklärt.

- Die Funktionsstörungen - oftmals bei schwereren Verlaufsformen - sind durch häufige Komorbiditäten kompliziert, wie z. B. Angststörungen, Depressionen aber auch somatische Erkrankungen. Bei der Entstehung der Erkrankungen spielen biologische, soziale und psychische Faktoren eine große Rolle. Auch die (Mikrobiom)-Darm-GehirnAchse kann Störungen aufweisen, die zu einer somatoformen autonomen Funktionsstörung des Verdauungssystems beitragen können.

- Die Diagnostik beinhaltet die ausführliche Anamnese, eine körperliche Untersuchung, ein Basislabor, eine Sonografie, eine gynäkologische Untersuchung, Endoskopien und ggf. speziellere Tests.

- Wichtig für ein gutes therapeutisches Ergebnis sind die klare Darstellung des Krankheitskonzeptes und eine gute Arzt-Patienten-Beziehung. Es ist wichtig, die weiteren Therapieschritte gemeinsam zu entscheiden, da es eine Vielzahl von therapeutischen Möglichkeiten gibt. Dazu gehören die medikamentöse Therapie und diätetische Maßnahmen, aber auch - v. a. bei schwereren Verlaufsformen Psychotherapie (kognitive Verhaltenstherapie, psychodynamische Therapie, Hypnotherapie).

\section{Interessenkonflikt}

Die Autoren geben an, dass keine Interessenkonflikte bestehen.

\section{Autorinnen / Autoren}

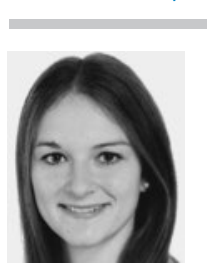

\section{Larissa Hetterich}

Seit 2015 Studentin der Humanmedizin an der Eberhard-Karls-Universität Tübingen, seit 2018 Mitarbeit und Dissertation in der AG somatoforme Störungen unter Leitung von Prof. Dr. med. Andreas Stengel in der Inneren Medizin VI, Abteilung Psychosomatische Medizin und Psychotherapie am Universitätsklinikum Tübingen.

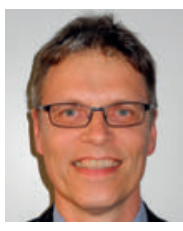

\section{Stephan Zipfel}

Prof. Dr. med. Studium der Humanmedizin in Heidelberg, London und Frankfurt am Main. Facharztausbildung für Innere Medizin und Psychosomatische Medizin und Psychotherapie, Ärztlicher Direktor der Abteilung Innere Medizin VI, Schwerpunkt Psychosomatische Medizin und Psychotherapie und Gesamtleitung der Psychoonkologie am CCC-Tumorzentrum Tübingen-Stuttgart.

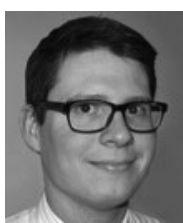

\section{Andreas Stengel}

Prof. Dr. med. Studium der Humanmedizin an der HU Berlin, 4-jähriger Forschungsaufenthalt an der UCLA in Los Angeles, Facharzt für Innere Medizin, Facharztausbildung für Psychosomatische Medizin an der Charité Berlin und am Universitätsklinikum Tübingen,

Arbeitsgruppenleiter in der Medizinischen Klinik m.S. Psychosomatik an der Charité Berlin und Oberarzt in der Inneren Medizin VI, Abteilung Psychosomatische Medizin und Psychotherapie, Leiter Ambulanz und Konsilwesen, Sektionsleiter Psychoonkologie.

\section{Korrespondenzadresse}

Prof. Dr. med. Andreas Stengel

Innere Medizin VI

Psychosomatische Medizin und Psychotherapie

Universitätsklinikum Tübingen

Osianderstr. 5

72076 Tübingen

E-Mail: andreas.stengel@med.uni-tuebingen.de

\section{Wissenschaftlich verantwortlich gemäß}

\section{Zertifizierungsbestimmungen}

Wissenschaftlich verantwortlich gemäß

Zertifizierungsbestimmungen für diesen Beitrag ist Prof. Dr. med. Andreas Stengel, Tübingen.

\section{Erstveröffentlichung}

Dieser Beitrag ist eine aktualisierte Version des Artikels: Hetterich L, Zipfel S, Stengel A. Gastrointestinale somatoforme Störungen. PSYCH up2date 2019; 13 (4): 327-340

\section{Literatur}

[1] Ronneberg C, Hausteiner-Wiehle C, Schäfert R et al. S3 Leitlinie „Funktionelle Körperbeschwerden“. AWMF online 2018; im Internet: https://www.awmf.org/uploads/tx_szlei tlinien/051-001I_S3_Funktionelle_Koerperbeschwer den_2018-11.pdf. Stand: 04.03.2019

[2] Wittkamp P, Andresen V, Broicher W et al. Prävalenz des Reizdarmsyndroms nach den Rom-III-Kriterien in Deutschland und Zusammenhänge mit potentiellen Risikofaktoren. Z Gastroenterol 2012; 50: V36

[3] Smith GR, Monson RA, Ray Dc. Psychiatric consultation in somatization disorder. N Engl J Med 1986; 314: 1407-1413

[4] Moser G. [Functional gastrointestinal disorders]. Wien Med Wochenschr 2006; 156: 435-440

[5] Luscombe FA. Health-related quality of life and associated psychosocial factors in irritable bowel syndrome: a review. Qual Life Res 2000; 9: 161-176

[6] Enck P, Azpiroz F, Boeckxstaens G et al. Functional dyspepsia. Nat Rev Dis Primers 2017; 3: 17081 
[7] Layer P, Andresen V, Pehl C et al. S3-Leitlinie Reizdarmsyndrom: Definition, Pathophysiologie, Diagnostik und Therapie. Gemeinsame Leitlinie der Deutschen Gesellschaft für Verdauungs- und Stoffwechselkrankheiten (DGVS) und der Deutschen Gesellschaft für Neurogastroenterologie und Motilität (DGNM). Z Gastroenterol 2011; 49: 237-239

[8] Enck P, Aziz Q, Barbara G et al. Irritable bowel syndrome. Nat Rev Dis Primers 2016; 2: 16014

[9] Hotz J, Madisch A, Enck P, Goebell H. Das Reizdarmsyndrom: Definition, Diagnosesicherung, Pathophysiologie und Therapiemöglichkeiten. Dtsch Arztebl International 2000; 97: A3263

[10] Henningsen P, Zipfel S, Herzog W. Management of functional somatic syndromes. Lancet 2007; 369: 946-955

[11] Creed F, Ratcliffe J, Fernandes L et al. Outcome in severe irritable bowel syndrome with and without accompanying depressive, panic and neurasthenic disorders. Br J Psychiatry 2005; 186: 507-515

[12] Moser G. Psychosomatik der Darmerkrankungen. Z Psychosomat Med Psychother 2006; 52: 112-126

[13] Soares RL. Irritable bowel syndrome: a clinical review. World ] Gastroenterol 2014; 20: 12144-12160

[14] Kinsinger SW. Cognitive-behavioral therapy for patients with irritable bowel syndrome: current insights. Psychology Res Behav Management 2017; 10: 231-237
[15] Van Oudenhove L, Crowell MD, Drossmann DA et al. Biopsychosocial aspects of functional gastrointestinal disorders. Gastroenterology 2016; pii: S0016-5085(16)00218-3. doi:10.1053/j.gastro.2016.02.027 [Epub ahead of print]

[16] Svedlund J, Sjödin I, Ottoson JO, Dotevall G. Controlled study of psychotherapy in irritable bowel syndrome. Lancet 1983; 2: 589-592

[17] Ford AC, Lacy BE, Harris LA et al. Effect of antidepressants and psychological therapies in irritable bowel syndrome: An updated systematic review and meta-analysis. Am J Gastroenterol 2019; 114: 21-39

[18] Peters SL, Muir JG, Gibson PR. Review article: gut-directed hypnotherapy in the management of irritable bowel syndrome and inflammatory bowel disease. Aliment Pharmacol Ther 2015; 41: 1104-1115

Bibliografie

DOI https://doi.org/10.1055/a-0996-0384 Fortschr Neurol Psychiatr 2019; 87: 512-525 (C) Georg Thieme Verlag KG Stuttgart · New York ISSN 0720-4299 


\section{Punkte sammeln auf CME.thieme.de}

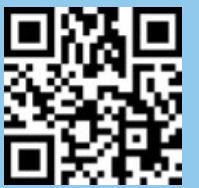

Diese Fortbildungseinheit ist in der Regel 12 Monate online für die Teilnahme verfügbar.

Den genauen Einsendeschluss finden Sie unter https://eref.thieme.de/CXDQGAK.

Sollten Sie Fragen zur Online-Teilnahme haben, finden Sie unter https://cme.thieme.de/hilfe eine ausführliche Anleitung. Wir wünschen viel Erfolg beim Beantworten der Fragen!

Unter https://eref.thieme.de/CXDQGAK oder über den QR-Code kommen Sie direkt zur Startseite des Wissenstests.

VNR 2760512019156642058

\section{Frage 1}

Welche Aussage trifft für die Diagnosestellung der funktionellen Dyspepsie zu? Die Diagnose der funktionellen Dyspepsie wird gestellt, wenn...

A postprandiales Völlegefühl, frühe Sättigung und epigastrische Schmerzen zusammen für 3 Monate auftreten.

B ein Patient entsprechende Risikofaktoren aufweist. Der Zeitraum spielt keine Rolle.

C postprandiales Völlegefühl, frühe Sättigung oder epigastrische Schmerzen 3 Monate anhalten und zwischen Symptombeginn und Diagnosestellung mindestens 6 Monate liegen.

D die Ösophago-Gastroduodenoskopie eindeutige Befunde aufweist.

E postprandiales Völlegefühl, frühe Sättigung oder epigastrische Schmerzen für mindestens 6 Monate durchgehend auftreten.

\section{Frage 2}

Welches Symptom dominiert beim postprandialen Distress-Syndrom?

A Schmerzen im Epigastrium während der Nahrungsaufnahme

B fehlender Appetit aufgrund durchgehender epigastrischer Schmerzen

C epigastrisches Brennen nach der Nahrungsaufnahme

D postprandiales Völlegefühl bzw. frühe Sättigung

E Erbrechen bei der Nahrungsaufnahme

\section{Frage 3}

Welchen der folgenden Subtypen gibt es nicht beim Reizdarmsyndrom?
A RDS-M: Misch-Subtyp
B RDS-D: Diarrhö-Subtyp
C RDS-C: Obstipations-Subtyp
D RDS-F: Flatulenz-Subtyp
E RDS-U: Unklassifizierter Subtyp

\section{Frage 4}

Welche Aussage trifft für somatoforme autonome Funktionsstörungen des Verdauungssystems zu?

A Somatoforme Komorbiditäten wie z. B. das Fibromyalgiesyndrom kommen sehr selten bei Patienten mit somatoformen autonomen Funktionsstörungen des Verdauungssystems vor.

B Patienten mit Reizdarmsyndrom haben zu 50-94\% psychische Komorbiditäten.

C Für den Verlauf und das Outcome der Therapie sind die Komorbiditäten irrelevant.

D Bei Patienten mit funktioneller Dyspepsie sind Essstörungen seltener anzutreffen als bei Patienten mit Reizdarmsyndrom.

E Bei Patienten mit somatoformen autonomen Funktionsstörungen treten oftmals nur psychische Komorbiditäten auf.

\section{Frage 5}

Welche folgenden Veränderungen sind nicht im Rahmen einer Störung der Darm-Gehirn-Achse zu erwarten?
A gestörte Darmmotilität
B verbesserte intestinale Permeabilität
C Änderung der intestinalen Immunantwort
D viszerale Hypersensitivität
E verändertes Mikrobiom

\section{Frage 6}

Welche der folgenden Methoden ist nach Empfehlung der deutschen Gesellschaft für Neurogastroenterologie und Motilität nicht zwingend notwendig zur Diagnostik der funktionellen Dyspepsie?

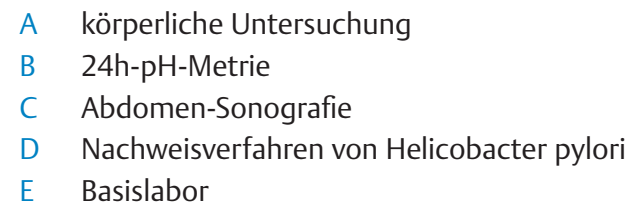




\section{Punkte sammeln auf CME.thieme.de}

Fortsetzung $\ldots$

\section{Frage 7}

Welche der folgenden Untersuchungen ist in der deutschen Leitlinie nicht zur Diagnosestellung des Reizdarmsyndroms vorgesehen?

A körperliche Untersuchung

B Abdomen-Sonografie

C gynäkologische Untersuchung

D explorative Laparoskopie

E obere und untere Endoskopie

\section{Frage 8}

Welche der folgenden Aussagen trifft nicht zu?

A Die Anamnese spielt eine wesentliche Rolle in der Diagnosefindung der somatoformen autonomen Funktionsstörungen.

B Bei somatoformen autonomen Funktionsstörungen ist es sehr wichtig, dass die Patienten das Krankheitskonzept verstehen.

C Nicht nur das Erfassen der körperlichen Symptome, sondern auch das Eruieren von belastenden Faktoren spielt eine wichtige Rolle.

D Wenn die Patienten weitere Diagnostik verlangen, sollte man dem Wunsch zum Vertrauensaufbau zwischen Arzt und Patient auf jeden Fall nachgehen.
E Die Diagnose einer somatoformen autonomen Funktionsstörung kann gestellt werden, wenn es keine organopathologischen Befunde gibt, die die Symptome des Patienten ausreichend erklären.

\section{Frage 9}

Welche der folgenden Therapiemöglichkeiten hat eine klare Empfehlung in der Behandlung somatoformer autonomer Funktionsstörungen des Verdauungssystems?
A Hypnotherapie
B Probiotika
C Stuhltransplantation
D glutenfreie Diät
E Heilfasten

\section{Frage 10}

\section{Welche Aussage zur Hypnotherapie trifft zu?}

A Diese ist sehr kostenintensiv und sollte nur Patienten mit schwersten Verlaufsformen vorbehalten bleiben.

B Diese sollte über mindestens ein Jahr durchgeführt werden.

C Diese kann unter Umständen auch kostensparend im Gruppensetting oder auch als mediengestützte Intervention erfolgen.

D Diese ist bislang kein anerkanntes Verfahren.

E Daten zur funktionellen Dyspepsie fehlen bislang. 